\title{
Automatic Aircraft Structural Topology Generation for Multidisciplinary Optimization and Weight Estimation
}

\author{
Mark D. Sensmeier* \\ Embry-Riddle Aeronautical University, Prescott, AZ 86314 \\ and \\ Jamshid A. Samareh ${ }^{\dagger}$ \\ NASA Langley Research Center, Hampton, VA 23681-2199
}

\begin{abstract}
An approach is proposed for the application of rapid generation of moderate-fidelity structural finite element models of air vehicle structures to allow more accurate weight estimation earlier in the vehicle design process. This should help to rapidly assess many structural layouts before the start of the preliminary design phase and eliminate weight penalties imposed when actual structure weights exceed those estimated during conceptual design. By defining the structural topology in a fully parametric manner, the structure can be mapped to arbitrary vehicle configurations being considered during conceptual design optimization. A demonstration of this process is shown for two sample aircraft wing designs.
\end{abstract}

\section{Introduction}

$\mathrm{M}$ ULTIDISCIPLINARY optimization and its application to the aero-structural design of aerospace vehicles has been a major research thrust in recent years. While much progress has been made in the ability to rapidly and automatically generate computational fluid grids to assess different external shapes based on parametric designs, the structural aspects of the design optimization problem have not enjoyed the same attention. In the few instances where structural layout is allowed to vary, the model used to analyze and assess candidate designs is usually overly simplistic and suspect from an accuracy perspective. Aircraft manufacturers have very accurate structural models of existing vehicles, but these models take literally months to generate and are not suitable for rapid consideration of candidate designs. Thus, there is a need for a methodology to create structural models of reasonably high accuracy while incorporating the ability to generate these models automatically for arbitrary external vehicle shapes.

As a first step towards developing this automatic model generation capability, an abstraction of the aircraft structural elements and their layout was constructed so that models can be created quickly for a given structural layout regardless of changes in external shape. Based on this abstraction, some initial algorithms to allow the mapping of parametric structural topologies to arbitrary wing shapes have been developed. These algorithms allow a structural designer to specify a structural layout in parametric terms, specifying locations in percentages of span and chord rather than actual dimensions. This specification is independent of the aircraft geometry so that upon making a change to the external vehicle shape, finite element models are automatically generated to allow sizing optimization to be performed. This process could then be automated to generate more accurate weight estimates for new aircraft concepts and to facilitate improved aerostructural optimization during the conceptual/preliminary design stages.

\section{Conceptual and Preliminary Design Processes}

The ability to generate structural layout and associated finite element models automatically will facilitate the incorporation of multidisciplinary optimization into the conceptual and preliminary design phases. Figure 1 shows a schematic representation of the conventional conceptual and preliminary design phases. Through tools like

\footnotetext{
* Assistant Professor, Aerospace Engineering Dept., 3700 Willow Creek Rd., Prescott, AZ 86301, (928)777-3847, mark.sensmeier@erau.edu, Senior Member AIAA.

† Senior Research Scientist, Multidisciplinary Optimization Branch, MS159, Hampton, VA 23681-2199, (757-8645776, jamshid.a.samareh@nasa.gov, Associate Fellow AIAA.
} 
FLOPS $^{1}$, conceptual design has come to include an optimization of aircraft vehicle configuration. However, structural design is generally not addressed to any significant extent in conceptual design. Vehicle weights are estimated as a function of the configuration parameters using historical or generic analysis-based data. The actual structural design is typically not selected until the preliminary design phase. If the aircraft being designed is not radically different from those on which the weights database is constructed, the designed weights will be fairly close to the estimates obtained during the conceptual design. However, if the "new" configuration is substantially different or original, a significant weight uncertainty can be introduced. By this time in the design process, making notable changes to the aircraft configuration can be extremely difficult as these changes will affect many systems, not just structures. Not only could this weight uncertainty be avoided by bringing structural design into the earlier stages of design, but multidisciplinary optimization could be enabled as well allowing the designer to strike the best balance between the vehicle configuration and the vehicle weight.

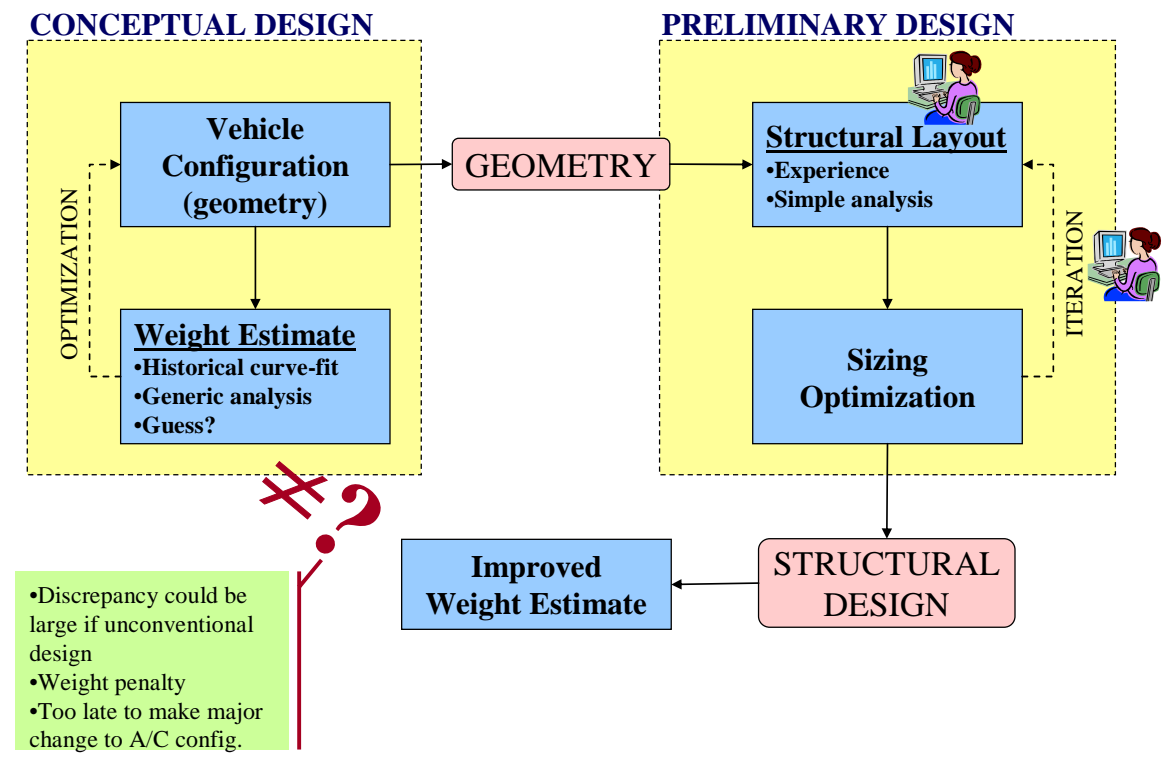

Figure 1. Schematic of convention conceptual and preliminary design processes.

In Fig. 2, an integrated approach to conceptual and preliminary design is illustrated. By automatically generating structural models for an arbitrary vehicle configuration, optimization of the structure can be incorporated into the conceptual/preliminary design phase. This will result in more accurate weight estimates for candidate vehicles and allow for multidisciplinary optimization to be conducted much earlier in the design process than in current practice.

Several researchers have attempted to bring conceptual-level structural models into the design process. Kelm, et $\mathrm{al}^{2}$ used a variety of quantitative (though non-finite element) analyses to more accurately assess wing weight during conceptual design. Sexstone ${ }^{3}$ introduced ELAPS, an equivalent-plate modeling tool, into conceptual trade studies and found it to be an efficient means to generate mass properties at an early stage. Röhl ${ }^{4}$ demonstrated the use of automatically-generated finite element models for optimization of a high-speed civil transport, while Cerulli, et al. ${ }^{5}$ have automated this process for a particular family of Airbus transports. The objective of the present effort is to move towards development of automatic structural layout and finite element model-generation algorithms suitable for designing a wide variety of aircraft families, including revolutionary concepts.

A key requirement in the development of these algorithms is the construction of a "demarcation line" between the geometrical specification of the wing exterior and the parametric specification of the topology of internal structural elements. The designer should be able to specify a structural topology with no reference to any shape variables (e.g., inboard spar at 20\% chord), including any discontinuities in the shape. The algorithms which map the parametric topology to the geometry to produce a specific design can be used to generate a new design in response to an arbitrary change in the wing exterior shape with no further human interaction. Thus, the geometry itself becomes a "black box" which is tapped into by the mapping algorithm as needed to determine the specific location and orientation of each structural element. Once the mapping is complete, the three-dimensional structure can be converted into a computational domain (e.g. finite element mesh) and a multidisciplinary sizing optimization can be performed. Finally, an optimization loop can be wrapped around this entire process to optimize the wing 
shape while updating the structural layout at each step to achieve improved weight estimates and aeroelastic responses if required. This process is also shown in Fig. 2.

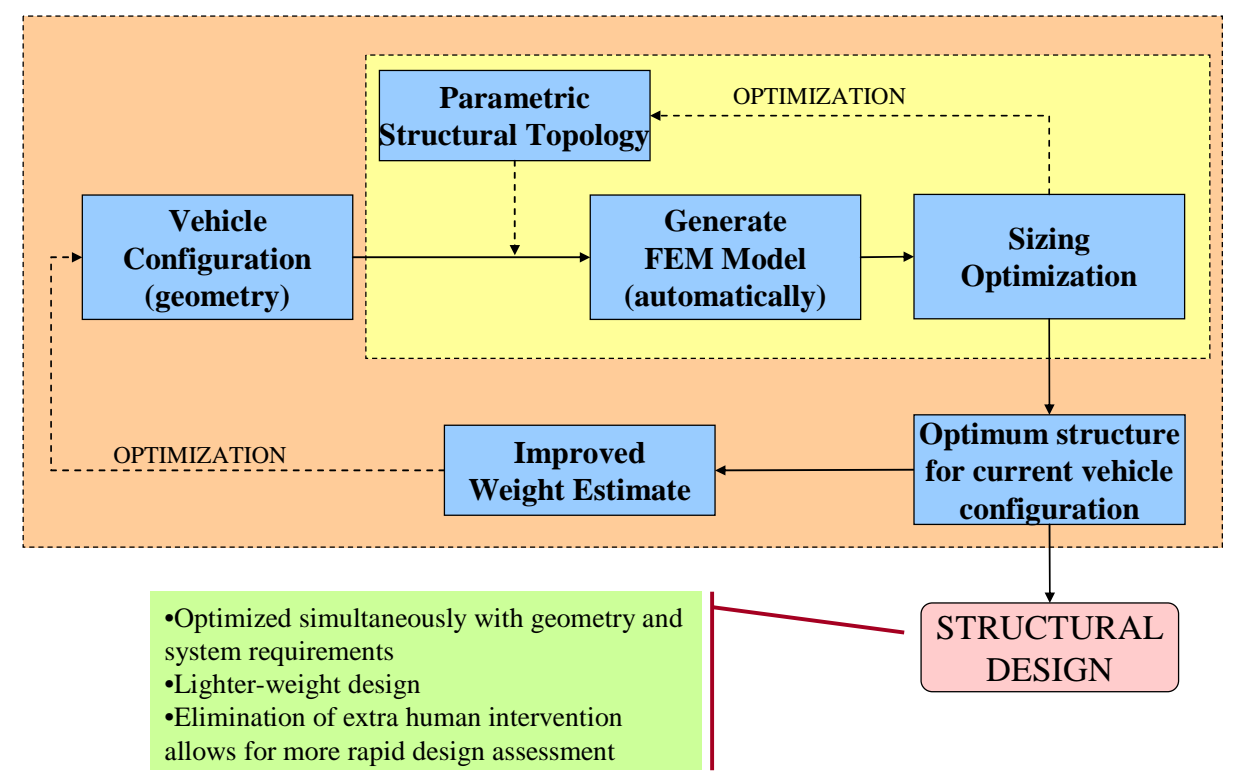

Figure 2. Proposed integration of conceptual and preliminary design with multi-level optimization

\section{Procedure}

In Figure 2, two embedded optimization loops were shown schematically. Although the structural optimization loop is shown as being nested within the overall vehicle system (geometry) optimization loop, these processes are more likely to be simultaneous in actual practice to enhance efficiency and perhaps avoid numerical difficulties. Though these optimizations are likely to be simultaneous, each requires some development to enable their application to the proposed vehicle design process. The present effort focuses on the structural optimization loop as shown in Fig. 3. The key to the success of this effort is the use of a fully parametric structural topology specification scheme which can easily be mapped onto an arbitrary vehicle geometry and structural models generated quickly and automatically.

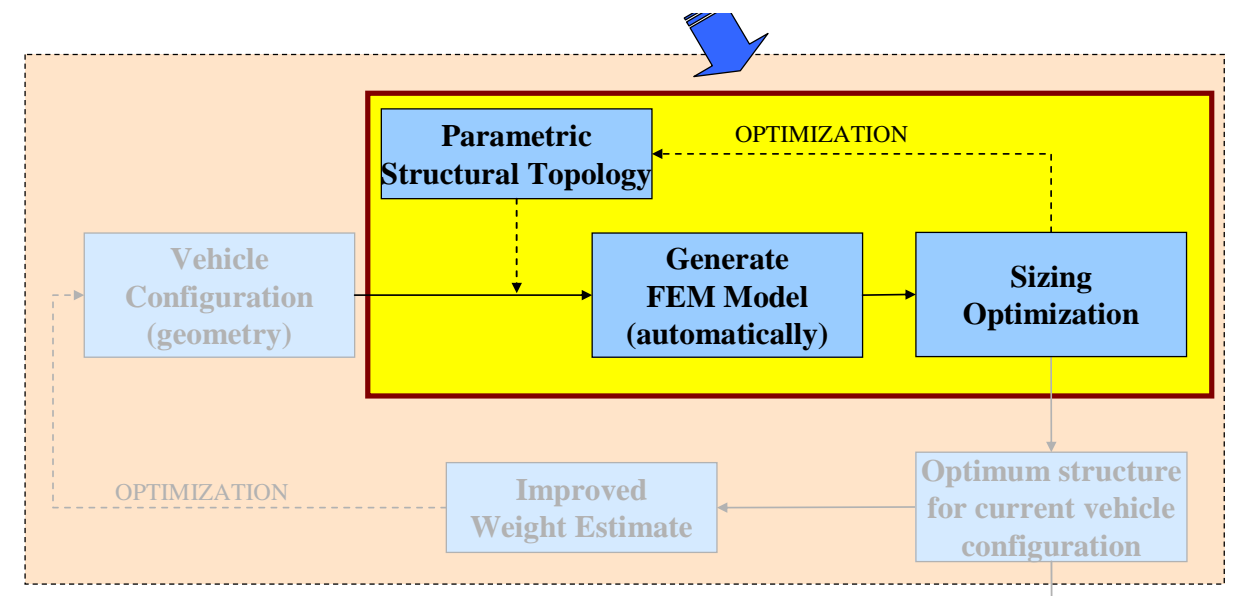

Figure 3. Structural optimization sub-loop 


\section{A. Demonstration Platform}

The ultimate product from this effort is a design-variable driven piece of software which takes the vehicle geometry and parametric structural topology as inputs and produces a finite element model as output. This model will then be sent to a commercial finite element analysis/optimization tool for sizing optimization. Ideally, this product should be platform-independent and present itself as a simple subroutine to the optimization control code.

A simple GUI-based tool, OptWing, was developed as a testbed in which to implement and evaluate candidate algorithms for mapping parametric structural elements to arbitrary wing configurations. This tool allows a user to specify the external shape of a wing and independently specify parametric structural component locations. The structural components can then be mapped to the current wing geometry. A finite element model of the mapped wing structure is then created and input files can be output to run either analysis of the wing or a sizing optimization. The user can choose one or more load case analyses to drive the optimization. This overall process is shown schematically in Figure 4. The "demarcation line" separating the Aircraft Configuration and Parametric Structural Layout emphasizes that the structural topology should be specified in such a manner that the structure can be mapped to any arbitrary wing geometry.

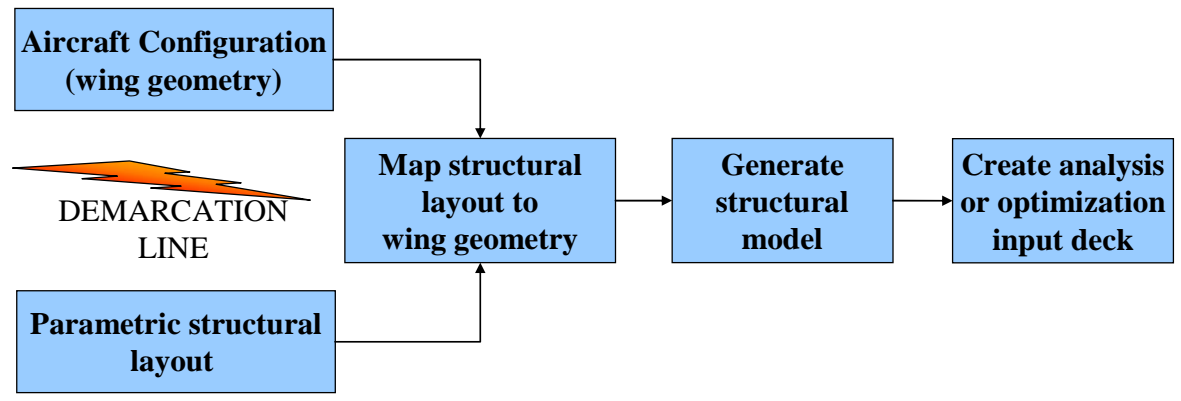

Figure 4. Schematic of model generation process.

\section{B. Wing Geometry}

For the purposes of the OptWing demonstration platform, a fairly simple geometry model is assumed. Wing boundaries (i.e. leading and trailing edges) are considered to be straight within a wing segment. Each segment may have a constant sweep angle, twist angle, and dihedral angle, taper and an independent span length. A wing may be built up from an arbitrary number of these straight segments. Currently, each wing is assumed to have a constant airfoil shape (though obviously the chord may differ at different spanwise locations). Figure 5 shows the OptWing Wing Geometry screen with a multi-segment wing specified.

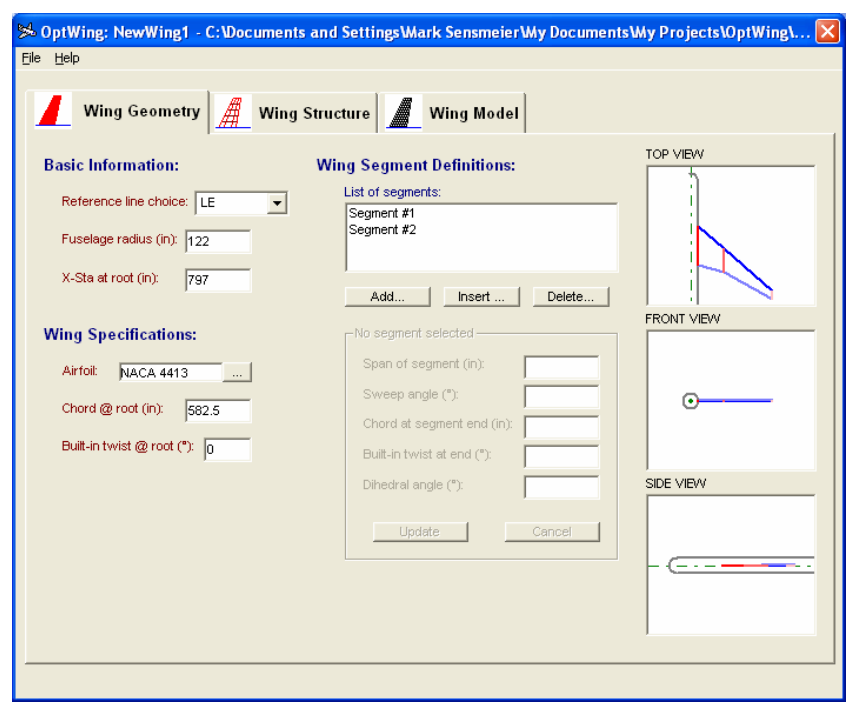

Figure 5. OptWing 'Wing Geometry’ screen showing multi-segment wing 
It is important to emphasize that the structural mapping algorithms and finite element mesh generation must be accomplished without any knowledge of the type of geometry model used. The geometry model must present itself as a "black-box" to the mapping algorithms and simply supply physical $(x, y, z)$ coordinates from input parametric information.

\section{Wing Parametric Coordinate System}

The simplest possible parametric coordinate system is used for the purpose of specifying the locations of structural components. This coordinate system is shown in Figure 6. The parametric coordinates are:

- $s$ spanwise coordinate ( 0 at root, 1 at tip)

- $\quad c$ chordwise coordinate ( 0 at local leading edge (LE), 1 at local trailing edge (TE))

- $\quad t$ thickness coordinate ( +1 at local airfoil top surface, -1 at local airfoil bottom surface)

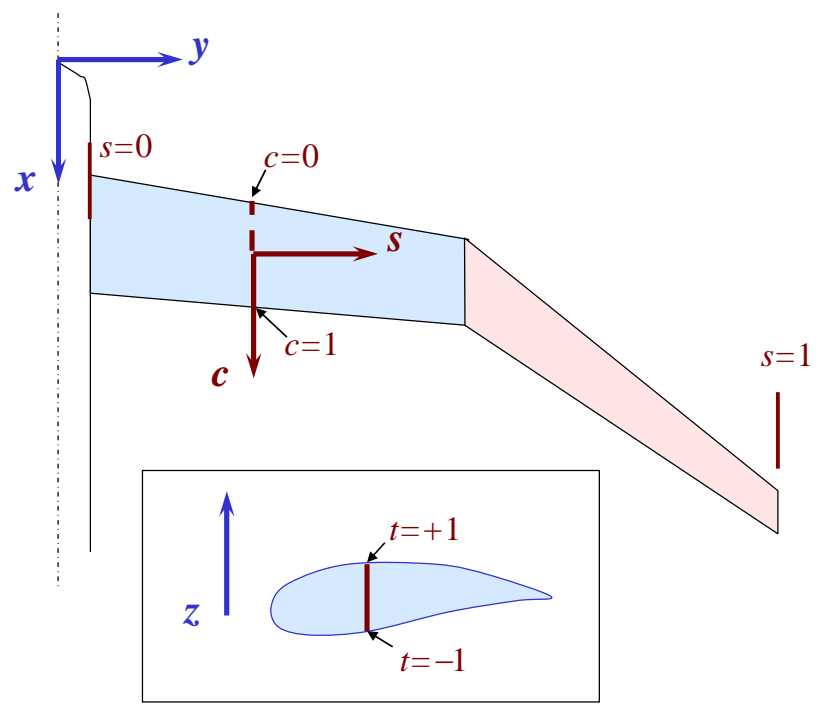

Figure 6. Parametric coordinate system used in OptWing

All location references for structural components must be linked to this parametric coordinate system or specified relative to other structural components. Thus, the locations and orientations must be stated in one of the following manners:

- Parametric coordinate locations (s, $c, t)$

- Locations relative to other structural elements (e.g. intersects at $s=0.3$ with nearest spar towards LE)

- Orientations relative to durable parametric geometric references (e.g. parallel or perpendicular to a specific $\%$ chord line)

- Orientations relative to other structural elements (e.g. perpendicular to LE spar)

\section{Wing Structure}

The structural components that make up a wing are assumed to be:

- $\quad$ Spars (webs and caps)

- Ribs

- Stringers

- $\quad$ Skin

The OptWing GUI contains three tabs: Wing Geometry, Wing Structure, and Wing Model. Within the Wing Structure tab on the main OptWing screen, the user can choose which type of structural components to define. The options for defining each of these structure elements are presented in this section. These are not intended to be exhaustive, merely representative of the typical vehicle structural layouts observed by Sensmeier \& Samareh ${ }^{6}$. 


\section{Spar Definitions}

Each spar in the wing is defined individually, and is assumed to be continuous from the top airfoil surface to the bottom airfoil surface. The presence of spar caps is implicitly assumed. The current options for spar definition include:

- Spar Inboard Location - The starting location of the spar is specified in terms of the parametric coordinates $(s, c)$.

- Spar Outboard Location - The spanwise parametric coordinate (s) at the point where the spar ends

- Spar Orientation - Several options are available to describe the spar orientation

o Constant \% of chord - Spar stays at the specified chordwise parametric coordinate (c) over its entire length

o Specified \% of chord - Spar follows a straight line path from its specified origin to its ending $(s, c)$ coordinates

o Joining nearest neighboring spar - Spar can be specified to end by joining the nearest neighbor towards either the LE or TE at the specified ending coordinate (s)

o Remain // to \% chord - Spar remains parallel to a given \% chord line over its entire length

\section{Rib Group Definitions}

Since most aircraft wings contain a large number of fairly evenly-spaced ribs, OptWing allows the user to specify groups of ribs. The current options for rib definition include:

- Span Range - The group of ribs can run the entire span of the wing or any subset, specified by the spanwise parametric coordinates $S_{\text {start }} S_{\text {end }}$.

- Span Reference line - This line defines the starting and ending points of a group of ribs. The first and last ribs within a group intersect the reference line at $s_{\text {start }}$ and $s_{\text {end }}$ and are evenly divided between these two points.

- Rib Extent - For most aircraft, the structural loading of the wing is primarily borne by the main wingbox (the structure between the LE and TE spars). This option allows the user to specify that the finite element model only represents the main wingbox. The user has the option, though, to specify the extent of a rib group to include the region from the LE spar to the LE of the wing and/or the main wingbox and/or the region from the TE spar to the TE of the wing.

- Rib Orientation - Currently, there are four options for the orientation of a group of ribs:

o Parallel to fuselage - All ribs in the group remain parallel to the fuselage centerline.

o Perpendicular to LE spar - All ribs in the group are perpendicular to the LE spar at the point where that particular rib intersects the LE spar

o Perpendicular to TE spar - All ribs in the group are perpendicular to the TE spar at the point where that particular rib intersects the TE spar

o Perpendicular to \% chord line - All ribs in the group are perpendicular to a specified local \% chord line.

- Rib Spacing - The number of ribs within a group is specified by one of two methods:

o The total number of rib divisions (so number of ribs equals number of divisions plus one)

o An approximate fixed spacing (the number of ribs is an integer which most closely matches the specified spacing). This is the only part of the parametric structural definition that involves a specific physical dimension. This capability was included to reflect the observation from the aircraft structural design trend study ${ }^{6}$ that most transport aircraft, regardless of size or wingspan, have the same rib spacing (approximately 24 inches).

\section{Stringer Definitions}

Stringers are not currently implemented in OptWing. Since stringers are represented in the finite element model as beam elements (as are the spar caps), the load transfer along the span of the wing is not accurately characterized unless the stringers are connected to skin elements (see below). The Stringer definition will be virtually identical to the Spar definition except for a flag specifying whether the stringer is on the top or bottom (or both) surface of the airfoil.

\section{Skin Definitions}

Skin regions are also not currently implemented in OptWing. We are currently working on implementing an appropriate meshing algorithm to generate the skin elements, which will be represented as shell elements. Since the skin regions are already defined by the presence and location of spars, ribs, and stringers, there is nothing for the 
user to define. However, each skin "panel" will most likely be associated with a flag to determine whether or not it is to be included in the finite element model.

\section{E. Wing Model}

The crux of the present effort is the development of mapping algorithms which take the fully parametric structural topology definition and map it to the current state of the wing geometry. Once this mapping is accomplished, a finite element mesh is created based on this mapping. From that mesh, different load cases may be applied and the commands to perform a structural analysis and/or sizing optimization may be generated. In the ultimate system, this would be invisibly within the overall vehicle configuration and structural optimization process. This process is manual within the OptWing testbed, as the user may make changes manually to the wing geometry and create the mapped model only for the current geometry.

The user has several options for output generated by Optwing:

- Mesh only - consists of only the nodes and elements.

- Analysis deck - consists of the nodes and elements, as well as the appropriate control cards to perform either a static (+ or $-\mathrm{g}$ ) wing loading, linear buckling analysis, or modal analysis.

- Sizing optimization deck - consists of the mesh, any load cases which the user specifies to include in the optimization, and the design variable, constraints, and fitness function (volume, or weight). For the optimization, the user can specify any subset of the structural components to be specified by design variables and/or constraints. The option screen in OptWing for optimization is shown in Fig. 7.

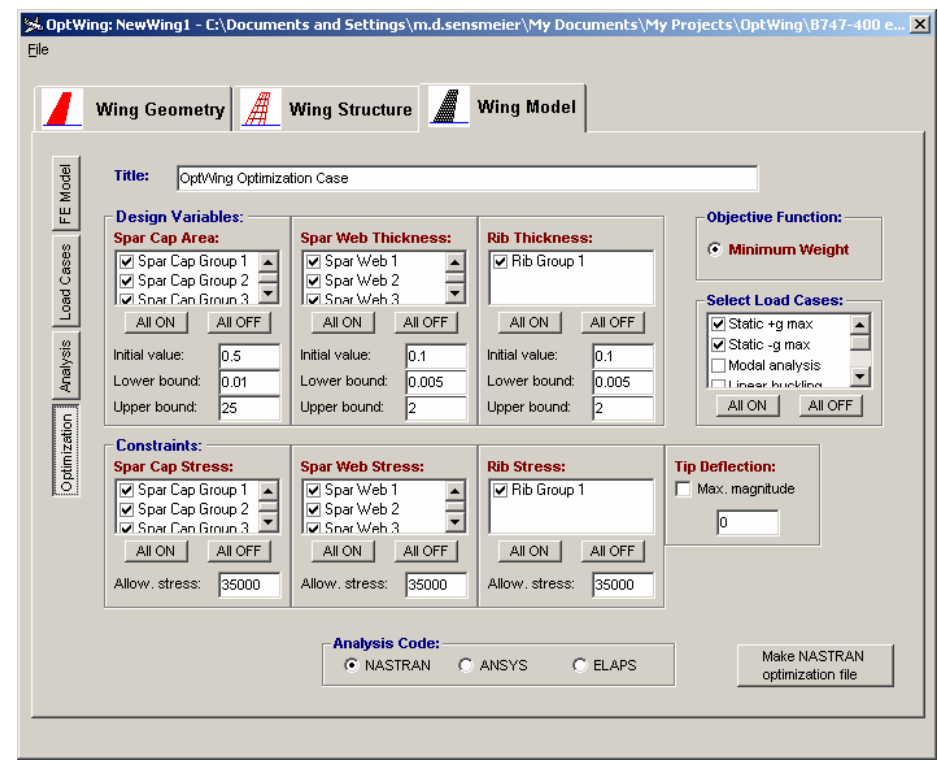

Figure 7. Sizing optimization options screen

Currently, OptWing will produce input decks for both ANSYS ${ }^{\circledR}$ and MSC/NASTRAN ${ }^{\circledR}$. The types of elements used for each component and analysis code is shown in Table 1.

Table 1. Element types used to generate finite element model

\begin{tabular}{|lcc|}
\hline Element Type & ANSYS $^{\circledR}$ & MSC/NASTRAN $^{\circledR}$ \\
\hline 3D Beam & BEAM4 & CBAR \\
\hline 3D Shell & SHELL63 & CQUAD4 \\
\hline
\end{tabular}

\section{Sample Applications}

To demonstrate how the current approach can be used to rapidly assess candidate vehicle configurations, the OptWing testbed was applied to two different types of aircraft wings:

- Boeing 747-400 commercial transport 
- NASA’s HALE (High-Altitude Long Endurance) aircraft

It is important to point out here that the results presented in this section are meant only to demonstrate the utility of the current approach in enhancing the conceptual/preliminary design phases. Since the current state of the tool does not include stringers and skin, actual analysis/optimization results are not realistic or representative of the actual aircraft. And although the cases shown in this section are generated individually with human intervention, the ultimate plan is to have these cases generated automatically as needed within the overall conceptual design optimization process.

\section{A. Boeing 747-400 Commercial Transport}

The Boeing 747-400 transport aircraft was selected as an example due to the easy availability of a cutaway diagram $^{7}$ to determine the actual structural layout and the presence of an "extra" spar near the wing root which joins its neighboring spar approximately one-third of the distance to the tip. The dimensions and a cutaway illustration of the 747-400 wing are shown in Fig. 8. The structure in the winglet was not modeled. The parametric structural topology was selected to approximately match the actual structure as illustrated in the cutaway. The components included in the model are shown in Table 2. The finite element mesh generated by OptWing is shown in Fig. 9.

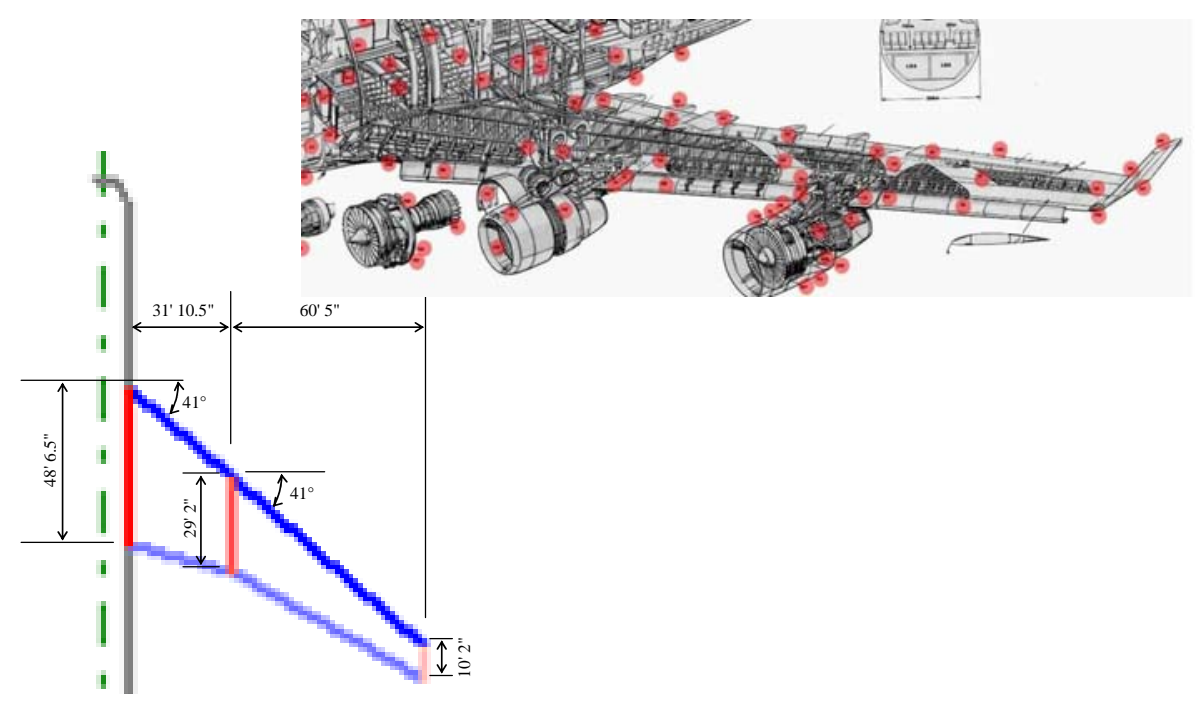

Figure 8. Dimensions and cutaway illustration of Boeing 747-400 wing

Table 2. Structural component definitions used in 747-400 example

\begin{tabular}{|c|c|c|}
\hline \multicolumn{2}{|c|}{ Spar Definitions } \\
\hline & From & To \\
\hline Spar \# & $s=0, c=0.35$ & $s=1, c=0.25$ \\
\hline 1 & $s=0, c=0.52$ & $s=0.63, c=0.58$ \\
\hline 2 & $s=0, c=0.68$ & $s=1, c=0.68$ \\
\hline 3 & $s=0, c=0.82$ & Jct. w/Spar\#3 at $s=0.3$ \\
\hline 4 & Rib Group Definition \\
\hline \multicolumn{3}{|c|}{ Full Line } \\
\hline \multicolumn{2}{|c|}{ Full span - main wingbox - 48 divisions- $\perp$ to LE spar - Ref. to TE spar } \\
\hline
\end{tabular}




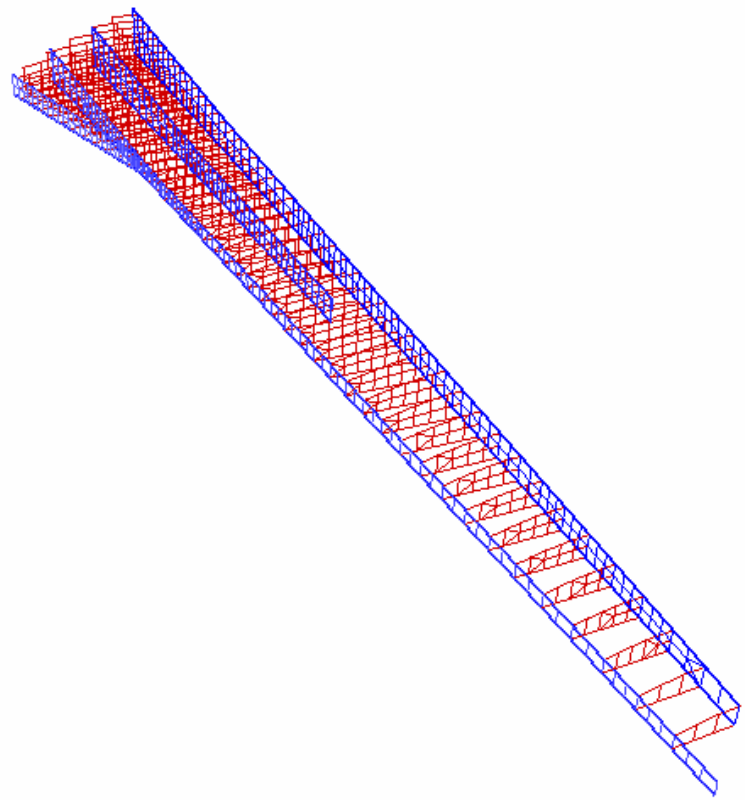

Figure 9. Finite element mesh generated for 747-400 sample problem

The optimization runs for the 747 wing were run in MSC/NASTRAN. For the baseline case, Fig. 10a shows the history of the wing weight during the optimization process. The history of the design variable values is shown in Fig. 10b.

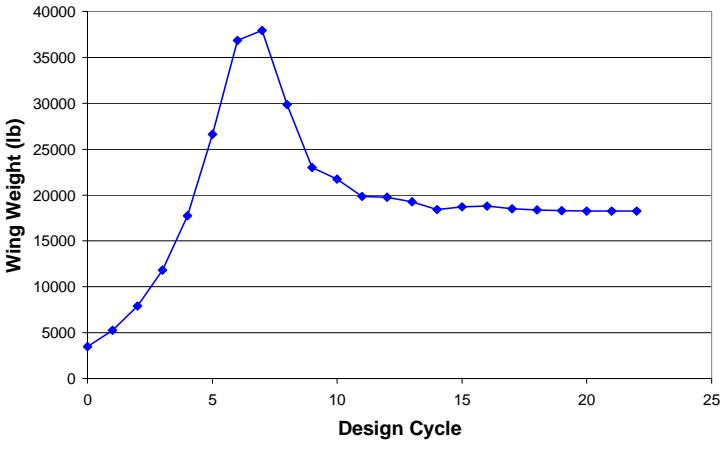

a) Wing weight history during optimization

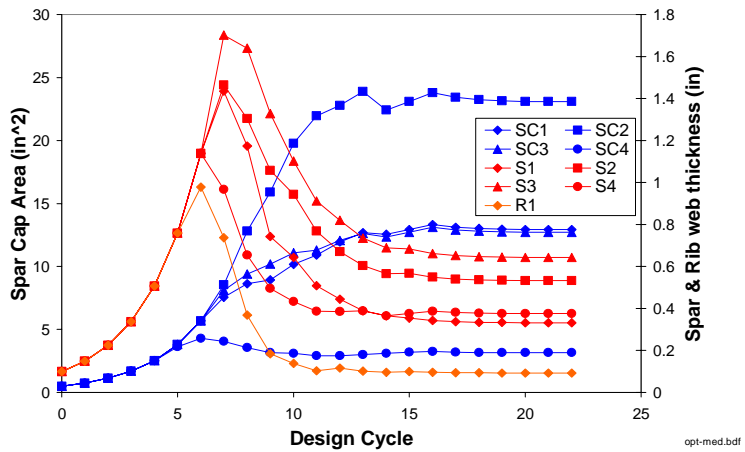

b) Design variable history during optimization

Figure 10. Baseline optimization results for 747-400 sample problem

One of the objectives of the present effort was to demonstrate how rapid model generation can be used to quickly estimate vehicle weight when changes are made to the geometry. To this end, two trade studies were accomplished, one where all wing dimensions were multiplied by a scale factor between 0.8 and 1.2, and one where the wing sweep angle was varied from $25^{\circ}$ to $55^{\circ}$. The results of these studies are shown in Fig. 11 . While these cases were submitted manually to NASTRAN, the ultimate objective would be for the overall conceptual/preliminary optimization control algorithm to call the model generator and then sizing optimization program as needed without human intervention.

\section{B. HALE (High-Altitude Long-Endurance) Aircraft}

The newest version of a High-Altitude Long Endurance (HALE) aircraft being studied by NASA was selected as a second example. A current representation of the geometry of the aircraft is shown in Fig. 12. Since the internal structure for this vehicle has not been fully defined, a two-spar arrangement was assumed. Additionally, the ability to input a custom airfoil was implemented so that the correct airfoil shape was used. The model generated by OptWing is shown in Fig. 13. 


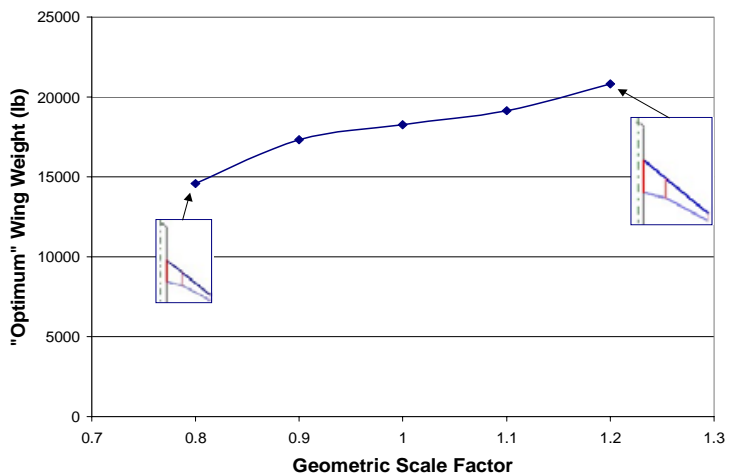

a) Effect of scale factor on optimum weight

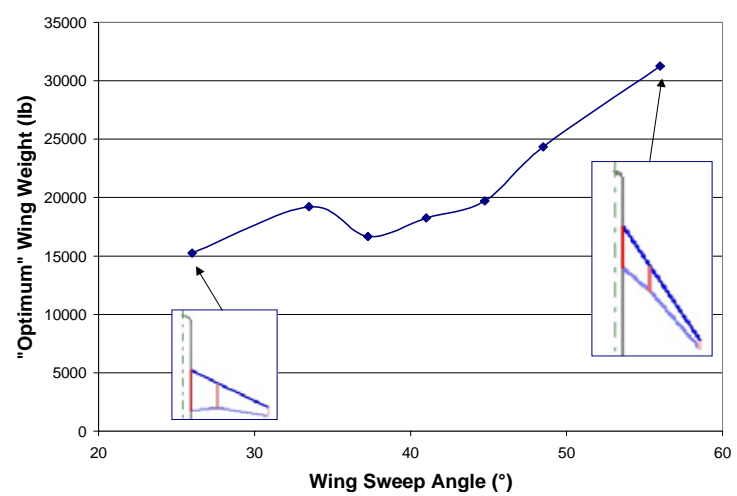

b) Effect of wing sweep angle on optimum weight

Figure 11. Demonstration of trade study capabilities using present approach.

The optimization cases for the HALE wing were run in ANSYS. For the baseline case, Fig. 14a shows the history of the wing volume (i.e. weight) during the optimization process. The history of the design variable values is shown in Figs. 14b and 14c.

A trade study was also performed for the HALE wing. In this case, though, the locations of the spars were varied. The LE spar was placed at $10 \%, 20 \%$, and $30 \%$ of chord and the TE spar at $60 \%, 70 \%$, and $80 \%$ of chord. The wing volume (weight) was optimized for all nine combinations of these locations. The results are shown in Fig. 15.

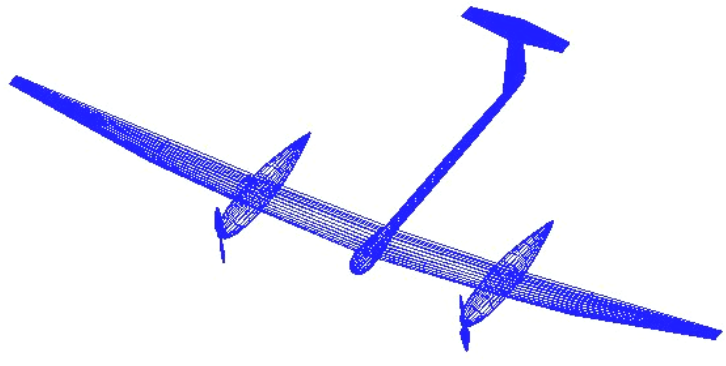

Figure 12. Geometry of proposed HALE vehicle

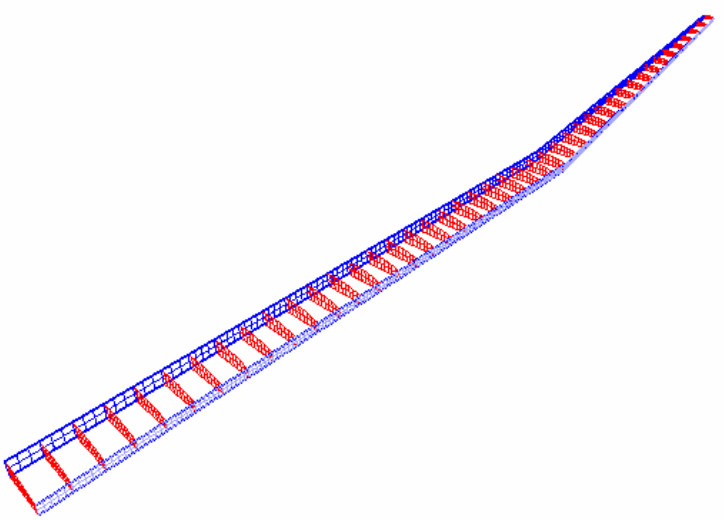

Figure 13. Finite element model for HALE wing

\section{Further Development}

An incremental development process is being used to add various capabilities to OptWing. Currently, efforts are underway to incorporate the skin and stringers into the finite element model. Other capabilities will be added eventually, including those related to both geometry/structure and analysis/optimization.

OptWing will continue to be developed as a testbed for the model generation algorithms. As these algorithms prove successful, they will be implemented into a batch, "black-box" version of model generation software. The batch version of the software must meet the following requirements:

- Be fully modular to allow easy modification

- The structural topology should be design-variable driven, not driven by user input.

- Must run on a variety of operating systems and platforms.

- Must access the wing geometry using a standard interface.

To meet these requirements, it was decided to implement the batch version using Python programming language, a multi-platform object-oriented open source language. 


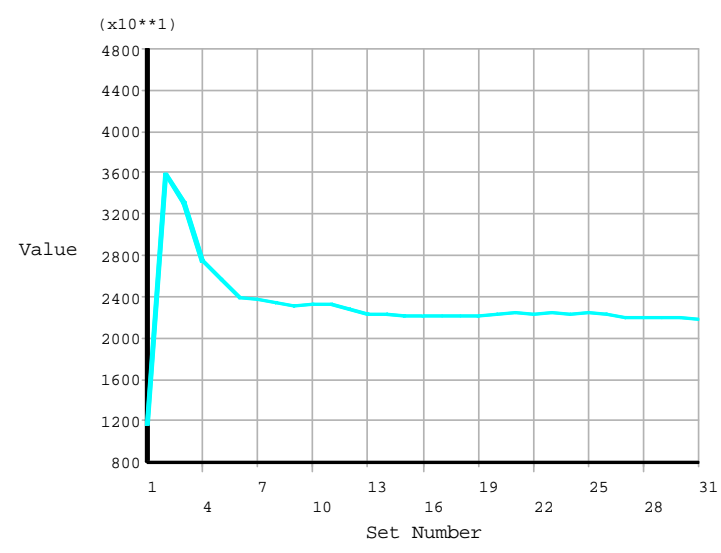

a) Wing volume (weight) history during optimization

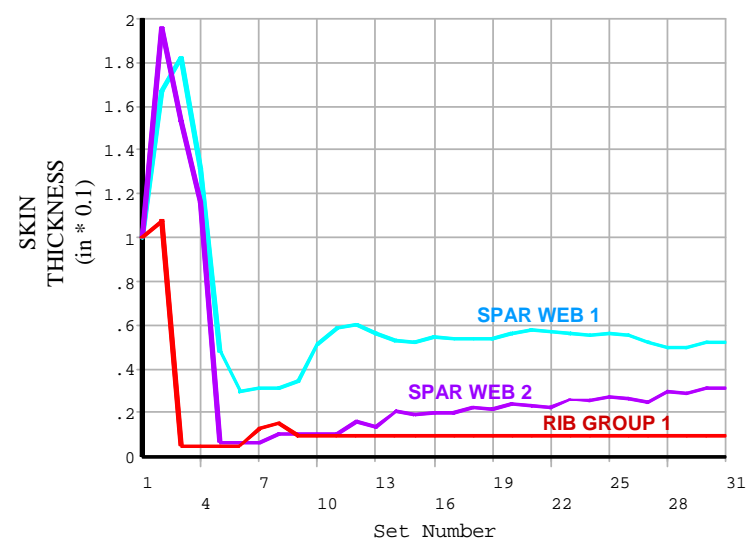

c) Spar web and rib thickness history during optimization

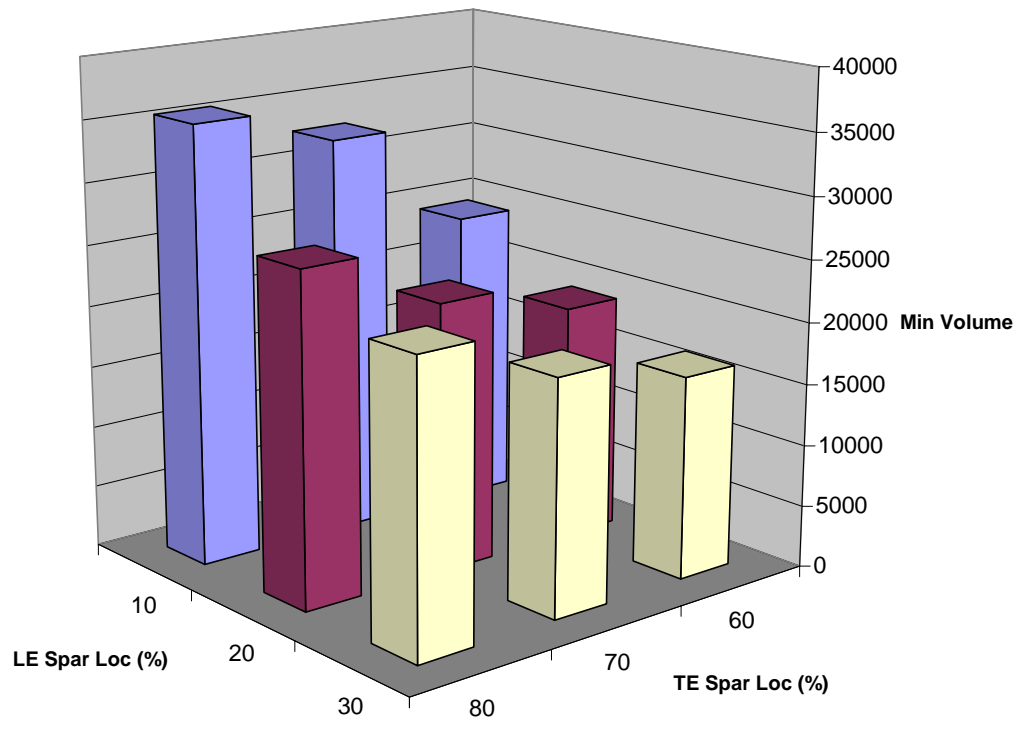

Figure 15. Demonstration of spar location trade study results for sample HALE problem 


\section{Conclusions}

The use of rapid model generation has been proposed as a means to improve the conventional approaches to conceptual and preliminary design of flight vehicles. By mapping a parametric description of the structural topology layout to a given vehicle geometry, a finite element model of moderate fidelity can be generated. This model can then be used within an optimization algorithm to determine the optimum structural dimensions. This allows for a more accurate weight estimate than one obtained from, say, curve-fits of historical data.

An initial software tool, OptWing, which accomplishes this mapping and rapid model generation has been developed and demonstrated for two different sample aircraft wings. This GUI-driven tool will serve as a prototype for a non-graphical implementation of these algorithms which can then be incorporated within the overall vehicle configuration optimization process to simultaneously optimize the aircraft geometry and structure.

\section{Acknowledgments}

The first author would like to gratefully acknowledge the support of NASA Langley Research Center on this research through Grant NNL04AA72G. The authors would also like to acknowledge the support of the NASA Faculty Fellows Program for its support of this effort during the summers of 2003 and 2004.

\section{References}

${ }^{1}$ McCullers, L. A., “Aircraft Configuration Optimization Including Optimized Flight Profiles, Multidisciplinary Analysis and Optimization Part I,” NASA CP-2327, 1984.

${ }^{2}$ Kelm, R., Läpple, M., and Grabietz, M., "Wing Primary Structure Weight Estimation of Transport Aircrafts in the Predevelopment Phase," $54^{\text {th }}$ Annual Conference of Society of Allied Weight Engineers, Huntsville, Alabama, SAWE Paper No. 2283, 22-24 May 1995.

${ }^{3}$ Sexstone, M. G., “Aircraft Structural Mass Property Prediction Using Conceptual-Level Structural Analysis,” 57 $7^{\text {th }}$ Annual Conference of the Society of Allied Weight Engineers, Wichita, Kansas, SAWE Paper No. 2410, 18-20 May 1998.

${ }^{4}$ Röhl, P. J., “A Multilevel Decomposition Procedure for the Preliminary Wing Design of a High Speed Civil Transport Aircraft,” Ph.D. Dissertation, School of Aerospace Engineering, Georgia Institute of Technology, Atlanta, GA, May 1995.

${ }^{5}$ Cerulli, C., Meijer, P. B., van Tooren, M. J. L., and Hofstee, J. W., "Parametric Modeling of Aircraft Families for Load Calculation Support," $45^{\text {th }}$ AIAA/ASME/ASCE/AHS/ASC Structures, Structural Dynamics \& Materials Conference, Palm Springs, California, AIAA Paper No. 2004-2019, 19-22 April 2004.

${ }^{6}$ Sensmeier, M. D. and Samareh, J. A., “A Study of Vehicle Structural Layouts in Post-WWII Aircraft," $45^{\text {th }}$ AIAA/ASME/ ASCE/AHS/ASC Structures, Structural Dynamics \& Materials Conference, Palm Springs, California, AIAA Paper No. 20041624, 19-22 April 2004. 